\title{
Perfiles diagnósticos y epidemiológicos en una U nidad de Memoria
}

\author{
Jorge González $\mathbf{H}^{1}$, Daniel Galdames $\mathrm{C}^{2}$, Sabrina 0 porto $\mathrm{S}^{3}$. \\ Diagnostic profiles at a Center for \\ Memory Disturbances
}

Background: The Memory Disturbances Center is a multidisciplinary team devoted to the study and treatment of patients with memory problems. Aim: To report the clinical features of patients consulting to this center. Material and methods: Retrospective review of the clinical records of the first 100 patients consulting in the center. Results: The age of consulting patients ranged from 17 to 93 years and 57 were female. The most common diagnoses were Alzheimer disease, depressive pseudo dementia, mild cognitive impairment, vascular dementia and adult attention deficit disorder. A formal neuropsychological assessment was performed in $60 \%$ and $20 \%$ entered a cognitive stimulation program. Conclusions: Although Alzheimer disease is the most common cause of memory disorders in the adult, concealed depression and attention deficit disorders are also common. A multidisciplinary approach allows a better diagnosis and treatment (Rev Méd Chile 2005; 133: 789-94).

(Key Words: Alzheimer disease; Alzheimer type senile dementia; Attention deficit disorder; Depression; Memory disorders)

Recibido el 20 de enero, 2004. Aceptado el 3 de mayo, 2005.

${ }^{1}$ Centro de Trastornos de la Memoria, Departamento de Neurología, Pontificia Universidad Católica de Chile. ${ }^{2}$ Hospital San Luis de Buin. ${ }^{3}$ Hospital de Urgencia de la Asistencia Pública, Pontificia Universidad Católica de Chile.

$\mathrm{E}^{\mathrm{n}}$ los últimos años, las neurociencias han experimentado un desarrollo exponencial, lo que ha permitido conocer con mayor profundidad el funcionamiento del cerebro normal y los mecanismos subyacentes a las enfermedades que lo afectan. Por otra parte, disponemos de nuevas técnicas de evaluación psicométrica y de neuroimágenes, impensables hace algunas décadas. Desde el punto de vista terapéutico, han apareci-

Correspondencia a: Dr. Jorge González H. Portugal \# 20, Depto. 48. Santiago, Chile. Fono/Fax: 6650385.

E-mail: joagonza@med.puc.cl do numerosas drogas psicotrópicas y técnicas de estimulación cognitiva que permiten tratar, con mayores probabilidades de éxito, a los pacientes con deterioro neuropsicológico.

El envejecimiento de la población y los cambios ambientales y de hábitos de vida propios de nuestra civilización, han favorecido un aumento importante en el número de pacientes con enfermedades cerebrales. En este sentido, las causas degenerativas, psiquiátricas, vasculares y traumáticas constituyen la mayor proporción.

Los elementos anteriormente descritos han llevado a la formación, en diferentes lugares del 
mundo, de centros especializados, constituidos por equipos multidisciplinarios, abocados específicamente al diagnóstico y rehabilitación de pacientes con trastornos cognitivos ${ }^{1}$.

El Centro de Trastornos de la Memoria (CETRAME), dependiente de la Pontificia Universidad Católica de Chile, inició sus funciones en julio de 2001. Está constituido por un equipo multidisciplinario de especialistas en neurología, psicología, fonoaudiología y terapia ocupacional. Es un centro abierto a la comunidad, particular, para adultos mayores de 15 años, con protocolos específicos de evaluación y estimulación neuropsicológica.

El objetivo de este artículo es describir las características de las primeras atenciones prestadas en el centro.

\section{Material y MÉTOdos}

En el Centro de Trastornos de la Memoria, el paciente consulta espontáneamente por problemas de memoria o es derivado para diagnóstico, rehabilitación cognitiva o ambos. En una primera instancia, es visto por el médico del equipo, el que, además de la anamnesis y examen físico, efectúa pruebas cognitivas y escalas de actividades de la vida diaria y de trastomos conductuales. Dentro de la evaluación, se considera un estudio de neuroimágenes y exámenes plasmáticos de rutina, incluyendo pruebas tiroideas y niveles de vitamina B12 y folatos.

Desde el punto de vista cognitivo, la evaluación inicial es con el Mini Mental Test de Folstein ${ }^{2}$. Esta prueba, aunque por sí misma no constituye diagnóstico, presenta ventajas que la hacen indispensable para una valoración de entrada del deterioro cognitivo. Es una herramienta breve, de uso universal, evalúa diferentes esferas de la cognición, su aplicación requiere un mínimo entrenamiento, la sensibilidad y especificidad es razonablemente alta y está normalizada para población hispana. Debe tenerse, eso sí, la precaución de utilizar normas de corrección adecuadas a la edad y escolaridad del paciente. El puntaje máximo es de 30 (mejor rendimiento). Cuando se requiere una medición psicométrica más específica, se utiliza la Batería Neuropsicológica para Hispanos ${ }^{3}$, además de otras pruebas si el caso lo amerita.

En Actividades de la Vida Diaria, AVD, se utiliza un cuestionario de 6 preguntas para activi- dades "básicas" (capacidad de ir al baño, vestirse, realizar su aseo personal, transferirse, controlar esfínteres y alimentarse $)^{4}$ y 8 para "instrumentales" (capacidad de llamar por teléfono, ir de compras, cocinar, lavar ropa, asear la casa, transportarse y manejar finanzas y fármacos) ${ }^{5}$. A mayor puntaje, mayor capacidad funcional.

Para evaluar trastornos conductuales se utiliza el Inventario Neuropsiquiátrico, NPI, que se aplica al cuidador. Evalúa la frecuencia y severidad de cada conducta (delirio, alucinaciones, agitación, depresión, ansiedad, euforia, apatía, desinhibición, irritabilidad, hipercinesia, sueño y apetito). A mayor puntaje, mayor alteración conductual ${ }^{6}$.

Para los diagnósticos de Alzheimer, depresión, demencia vascular (AVE) y trastorno por déficit atencional (TDA) se utilizó los criterios DSM IV ${ }^{7} \mathrm{y}$ para deterioro cognitivo leve (DCL), los de Peterson ${ }^{8}$.

Los criterios para Alzheimer consideran un deterioro que, además de incluir la memoria, afecte al menos otra función cognitiva, y que sea de magnitud suficiente para deteriorar la capacidad laboral o social del paciente. El curso debe ser lentamente progresivo y deben descartarse otras causas que puedan producir este deterioro, como delirium, depresión o trastornos metabólicos. Si la demencia es explicable por una enfermedad cerebrovascular subyacente, determinada por criterios clínicos o imagenológicos, entonces estamos frente a una demencia vascular?.

El DCL se define como un trastorno caracterizado por la pérdida aislada y objetiva de la memoria, sin compromiso de las actividades de la vida diaria ${ }^{8}$.

Para el diagnóstico de Episodio Depresivo Mayor, se requiere la presencia de ánimo depresivo o anhedonia, por al menos dos semanas, asociado a al menos cuatro de los siguientes síntomas: alteraciones del apetito, trastornos del sueño, agitación o enlentecimiento psicomotor, fatiga, sentimientos de culpa, quejas cognitivas e ideas de muerte ${ }^{7}$. Además de la anamnesis, el NPI es una excelente herramienta para la evaluación de estos síntomas por los familiares, lo cual es fundamental en el caso de pacientes con deterioro cognitivo, que presentan dificultad para recordar su estado anímico reciente ${ }^{6}$.

En el caso del TDA, el DSM IV exige criterios de desatención o hiperactividad-impulsividad, de ini- 
cio en la infancia y de magnitud suficiente para afectar el rendimiento social, académico o laboral ${ }^{7}$. En el CETRAME se utiliza la Adult Self Report Scale (OMS), para evaluar la severidad de los síntomas ${ }^{9}$.

La rehabilitación cognitiva se define como un programa integrado que incluye todos aquellos procedimientos dirigidos a la integración social y a la adaptación funcional del paciente con lesión 0 disfunción cerebral. Este programa se diseña individualmente de acuerdo a las necesidades de cada enfermo. En general son dos sesiones semanales, en que se realiza estimulación funcional, con trabajo de escritorio o por ordenador, terapia ocupacional, psicoterapia y educación al paciente y cuidador ${ }^{10,11}$.

Se analizó el total de fichas de pacientes que consultaron al CETRAME entre julio de $2001 \mathrm{y}$ agosto de 2002. No se incluyó los casos que llegaron para evaluación psicométrica o rehabilitación neuropsicológica, sólo como servicios de apoyo, y que no fueron examinados por algún médico del equipo.
Estadística. La información recopilada se procesó en el Programa Epi Info 2002, utilizándose la prueba de $t$ de student, para evaluar la significancia de las diferencias de promedios de edad, educación y puntajes para los diferentes diagnósticos, y la prueba de chi cuadrado para las diferencias por sexo. Se consideró un resultado como estadísticamente significativo cuando el valor de "p" fue menor a 0,05.

\section{RESULTADOS}

Se incluyó un total de 100 fichas. El promedio de edad de los pacientes fue de 61 años (rango 17 a 93), observándose un significativo porcentaje de pacientes adultos jóvenes (Figura 1). El 57\% de los pacientes fue de sexo femenino. El promedio de educación (años aprobados) fue de 14 años. Sesenta y dos por ciento de los pacientes tenía estudios superiores (Figura 2).

FIgURA 1. Distribución de pacientes según edad.

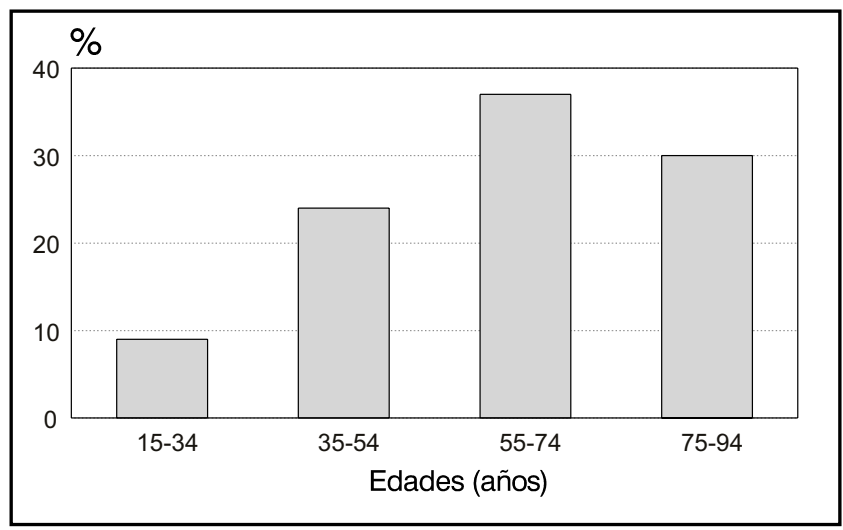

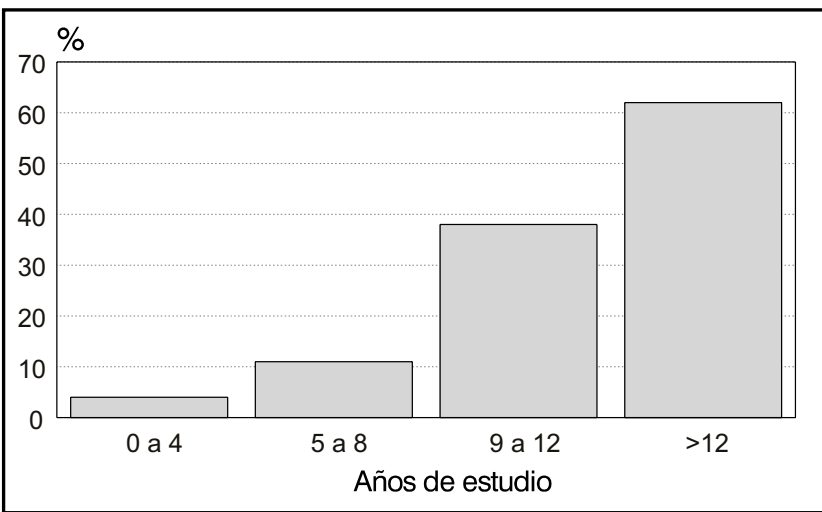

Figura 2. Distribución de pacientes según años de estudio. 


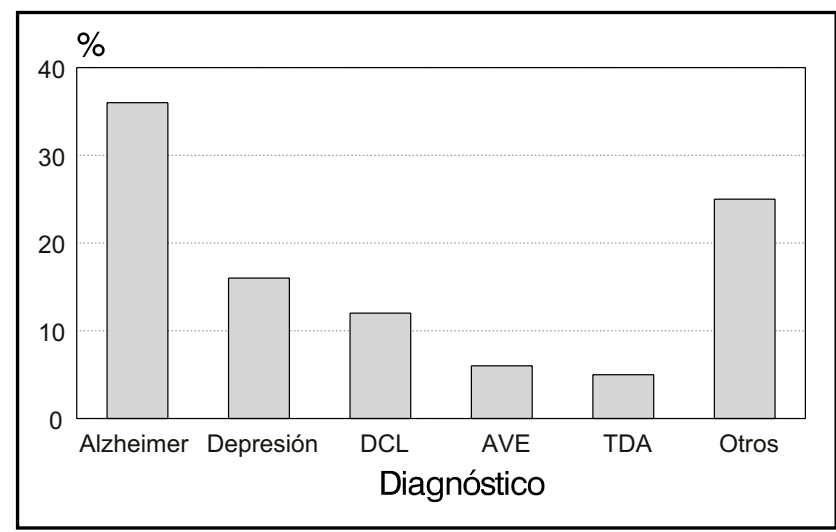

FIgURA 3. Distribución de pacientes según diagnósticos.

La Figura 3 muestra los diagnósticos más frecuentes presentados por los pacientes. En primer lugar aparece el diagnóstico de enfermedad de Alzheimer, seguido por pseudodemencia depresiva, deterioro cognitivo leve, demencia vascular y trastorno por déficit atencional, respectivamente. El grupo "otros" incluye cuadros metabólicos (especialmente tiroideos, hipoxia y déficit de B12), psiquiátricos (trastorno de ansiedad, esquizofrenia), infecciosos (encefalitis, Jacob Creutzfeldt) y trastornos postquirúrgicos.

La Tabla 1 muestra los promedios de edad, años de estudio y sexo para los diferentes diagnósticos. En la distribución por edad el mayor promedio lo alcanzó la enfermedad de Alzheimer, y los más jóvenes los pacientes con
TDA ( $p<0,05)$. La pseudodemencia depresiva, el DCL y la demencia vascular ocuparon un lugar intermedio.

Los promedios de años de estudio fueron similares en todos los grupos. Hubo una tendencia a mayor número de pacientes de sexo femenino para Alzheimer, depresión y TDA, sin alcanzar significancia estadística.

La Tabla 2 muestra los promedios de cognición (Mini Mental), conducta (NPI) y funcionalidad (AVD), para los diferentes diagnósticos. En cuanto a cognición, se aprecia un deterioro significativamente mayor en Alzheimer y AVE, con relativa preservación en Depresión, TDA y DCL $(p<0,05)$.

La conducta estaba más alterada en Alzheimer y AVE, siendo la alteración mínima para DCL ( $p$

Tabla 1. Promedios y desviaciones estándar de edad, educación y sexo para los diferentes diagnósticos

\begin{tabular}{|lccccc|}
\hline & Alzheimer & Depresión & DCL & AVE & TDA \\
\hline Edad (años) & $75,0(12,5)$ & $63,4(14,8)$ & $60,1(12,0)$ & $49,2(9,7)$ & $38,8(7,5)$ \\
Estudios (años) & $12,8(4,5)$ & $13,5(4,5)$ & $14,1(5,1)$ & $16,0(5,4)$ & $15,2(2,2)$ \\
Sexo femenino (\%) & 67 & 63 & 50 & 50 & 80 \\
$\mathrm{~N}^{\circ}$ de pacientes & 36 & 16 & 12 & 6 & 5 \\
\hline
\end{tabular}

Tabla 2. Promedios y desviaciones estándar de puntajes en tests y escalas al ingreso según diagnóstico

\begin{tabular}{|lccccc|}
\hline & Alzheimer & Depresión & DCL & AVE & TDA \\
\hline Mini Mental & $19,0(6,5)$ & $27,6(4,1)$ & $28,8(1,3)$ & $19,7(10,1)$ & $29,0(2,0)$ \\
NPI & $24,9(14,5)$ & $16,0(10,4)$ & $4,5(3,9)$ & $29,0(8,2)$ & - \\
AVD básicas & $5,5(1,0)$ & $6,0(0,0)$ & $5,9(0,3)$ & $4,3(2,3)$ & $6,0(0,0)$ \\
AVD & $3,8(2,9)$ & $7,8(1,0)$ & $7,9(0,3)$ & $2,5(3,6)$ & $8,0(0,0)$ \\
instrumental & & & & & \\
\hline
\end{tabular}




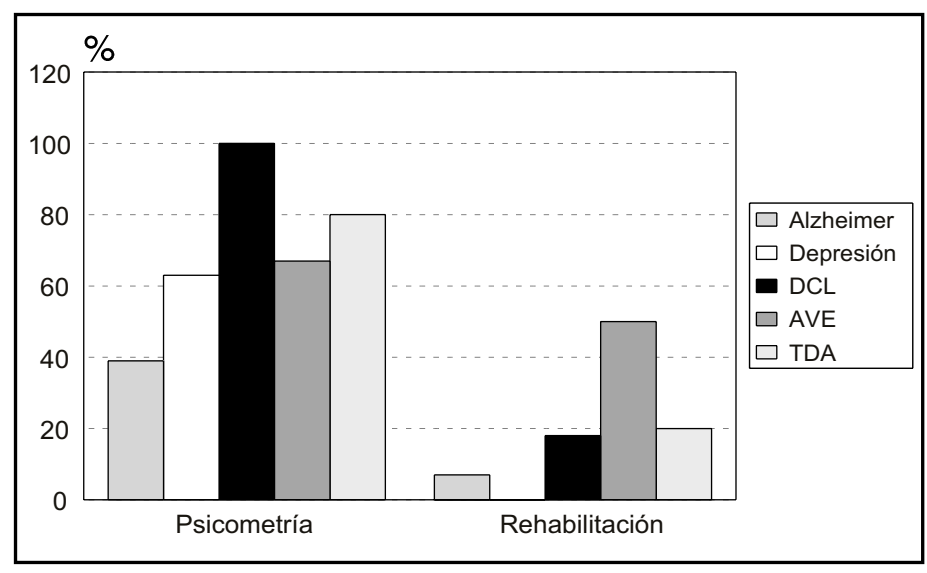

Figura 4. Frecuencia de evaluación psicométrica y rehabilitación cognitiva, para los diferentes diagnósticos.

$<0,05)$. No se aplicó el NPI a pacientes con TDA ya que éstos habitualmente no acuden con acompañante.

Las Actividades Básicas de la Vida Diaria estaban en general, preservadas, excepto para las demencias vasculares, en que hubo también un deterioro físico neurológico relevante (ns). Las Actividades Instrumentales de la Vida Diaria estaban significativamente más alteradas en Alzheimer y demencia vascular $(p<0,05)$.

La Figura 4 muestra la frecuencia de evaluación psicométrica formal e ingreso a un programa de rehabilitación cognitiva para los diferentes diagnósticos. En el total de los pacientes con DCL se realizó una evaluación psicométrica, examen imprescindible para este diagnóstico. También, se realizó frecuentemente este examen en pacientes con AVE (planificación de rehabilitación), TDA y depresión (diagnóstico diferencial). En la mayoría de los pacientes con Alzheimer fue suficiente sólo el Mini Mental Test y la evaluación clínica para el diagnóstico. La escasa proporción de pacientes que ingresan a programas de rehabilitación cognitiva está dada principalmente por factores de tipo económico y de dificultad en el traslado, que muchas veces debe hacerlo el cuidador.

\section{DisCUSIÓN}

Los datos previamente expuestos nos permiten establecer las características de los pacientes que acudieron a la clínica de la memoria.
Los diagnósticos más frecuentes fueron Alzheimer, depresión y deterioro cognitivo Leve, respectivamente. Estos datos enfatizan la importancia de buscar dirigidamente síntomas depresivos en pacientes que consultan por problemas de memoria y la necesidad de una evaluación psicométrica formal a aquellos pacientes en que la evaluación cognitiva general sea normal. De otra forma, catalogaremos como sanos a una gran cantidad de pacientes que caen en la categoría de DCL, entidad definida como pre-Alzheimer, potencialmente recuperable ${ }^{12}$.

Contrario a lo que pudiera esperarse, una proporción importante de los pacientes fueron adultos jóvenes. Entre estos últimos, predominan enfermedades como el TDA y la demencia vascular. El trastorno por déficit atencional del adulto es una enfermedad frecuente, invalidante y tratable. Se requiere un alto índice de sospecha para el diagnóstico ${ }^{9}$.

Los deterioros cognitivos de origen vascular, son potencialmente recuperables con terapias de estimulación cognitiva, la cual debe iniciarse a la brevedad $^{13}$.

A continuación se describe el perfil clínico observado en este estudio, para las patologías más prevalentes: En el caso del Alzheimer, se trata de pacientes de aproximadamente 75 años, nivel cognitivo general deteriorado, significativa alteración conductual y afectación de las actividades instrumentales de la vida diaria. La pseudodemencia depresiva presenta un promedio de 65 años de edad, el nivel cognitivo general está preservado, 
se observa trastornos conductuales en el sentido de ideas depresivas, apatía y trastornos del sueño, con conservación de las actividades de la vida diaria.

En el deterioro cognitivo leve, el promedio de edad es de 60 años y hay una preservación del nivel cognitivo general, las actividades de la vida diaria y la conducta. Las alteraciones cognitivas sólo son evidenciables a la psicometría formal.

Las demencias vasculares presentan un promedio de edad de 50 años, con significativo deterioro cognitivo general, de la conducta y de

\section{REFERENCIAS}

1. López-Pousa S, Serra-Mestres J, Lozano-Galeggo M, Tron-Estrada A, Vilalta-Franch J, Garre-Olmo J ET AL. Programa Integral de Atención Psicogeriátrica. Rev Neurol 2000; 30: 1181-7.

2. Folstein M, Folstein S, Mchugh P. "Mini Mental State" a practical method for grading the cognitive state of patients for the clinician. J Psychiatr Res 1975; 12: 189-98.

3. Pontón M, Sats P, Herrera L, Ortiz F, Urrutia C, Young R ET AL. Normative data stratified by age and education for the Neuropsychological Screening Battery for Hispanics (NeSBHIS): Initial report. JINS 1996; 2: 96-104.

4. Katz S, Ford A, Moskowitz R. Studies of illness in the aged. The index of ADL: a standardized measure of biological and psychosocial function. JAMA 1963; 185: 914-9.

5. Lawton M, Brody E. Assessment of older people: self-maintaining activities of daily living. Gerontologist 1969; 9: 179-86.

6. Cummings J, Mega M, Gray K, Rosenberg-ThompSON S, Carusi D, Gornbey J. The neuropsychiatric inventory: comprehensive assessment of psy- las actividades básicas e instrumentales de la vida diaria.

Los pacientes con déficit atencional del adulto tienen una media de 40 años. El nivel cognitivo general y las actividades de la vida diaria están preservados. El diagnóstico se apoya especialmente en la historia remota y la presencia de elementos clínicos y anamnésicos de inatención o impulsividad-hiperactividad.

Es de notar el limitado acceso que tienen los pacientes a los programas de estimulación cognitiva, lo cual podría mejorarse estableciendo paquetes de rehabilitación a menores costos e implementando protocolos de estimulación domiciliarios.

chopathology in dementia. Neurology 1994; 44: 2308-14.

7. American Psychiatric Association. Diagnostic and Statistical Manual of Mental Disorders. $4^{\text {th }}$ ed. Washington, DC: American Psychiatric Association, 1994.

8. Petersen R, Doody R, Kurz A, Mohs R, Morris J, Rabins P et al. Current Concepts in Mild Cognitive Impairment. Arch Neurol 2001; 58: 1985-92.

9. Murphy K, AdLeR L. Assessing Attention-Deficit/ Hyperactivity Disorder in Adults: Focus on Rating Scales. J Clin Psychiatry 2004; 65 [suppl 3]: 12-7.

10. FernándeZ-GuinEA S. Estrategias a seguir en el diseño de los programas de rehabilitación neuropsicológica para personas con daño cerebral. Rev Neurol 2001; 33: 373-7.

11. López-Luengo B. Orientaciones en rehabilitación cognitiva. Rev Neurol 2001; 33: 383-7.

12. Shand B, González J. Deterioro cognitivo leve primer paso a la demencia? Cuad Neurol 2003; 27: 31-8.

13. Cicerone $K$, Dahlberg C, Kalmar $K$, Langenbahn D, Malec J, Bergquist T et al. Evidence-Based Cognitive Rehabilitation: Recommendations for Clinical Practice. Arch Phys Med Rehabil 2000; 81: 1596-615. 